\title{
Efficacy and Limitations of Transarterial Acrylic Glue Embolization for Intracranial Dural Arteriovenous Fistulas
}

\author{
Naoko MiYamoto, ${ }^{1}$ Isao NAito, ${ }^{1}$ Tatsuya SHIMizU, ${ }^{2}$ and Yuhei YoshimotO ${ }^{2}$ \\ ${ }^{1}$ Department of Neurosurgery, Geriatrics Research Institute and Hospital, Maebashi, Gunma; \\ ${ }^{2}$ Department of Neurosurgery, Gunma University Graduate School of Medicine, \\ Maebashi, Gunma
}

\begin{abstract}
The efficacy and limitations of transarterial acrylic glue embolization for the treatment of intracranial dural arteriovenous fistulas (DAVFs) were investigated. Thirty-four DAVFs treated by transarterial embolization using n-butyl cyanoacrylate were retrospectively reviewed. The locations of DAVFs were the transverse-sigmoid sinus in 11, tentorium in 10, cranial vault in 9, and superior sagittal sinus, jugular bulb, foramen magnum, and middle cranial fossa in 1 each. Borden classification was type $I$ in 7 , type II in 3, and type III in 24. Eight patients had undergone prior transvenous coil embolization. Complete obliteration rate was $56 \%$ immediately after embolization, $71 \%$ at follow-up angiography, and $85 \%$ after additional treatments ( 1 transvenous embolization and 4 direct surgery). Complications occurred in three patients, consisting of asymptomatic vessel perforations during cannulation in two patients and leakage of contrast medium resulting in medullary infarction in one patient. Transarterial glue embolization is highly effective for Borden type III DAVF with direct cortical venous drainage, but has limitations for Borden type I and II DAVFs in which the affected sinus is part of the normal venous circulation. Onyx is a new liquid embolic material and is becoming the treatment of choice for DAVF. The benefits of glue embolization compared to Onyx embolization are high thrombogenicity, and relatively low risks of cranial nerve palsies and of excessive migration into the draining veins of high flow fistula. Transarterial glue embolization continues to be useful for selected patients, and complete cure can be expected in most patients with fewer complications if combined with transvenous embolization or direct surgery.
\end{abstract}

Key words: acrylic glue, dural arteriovenous fistula, embolization

\section{Introduction}

The natural history of intracranial dural arteriovenous fistulas (DAVFs) depends on the venous drainage pattern. DAVFs with cortical venous reflux (CVR) carry a high risk of hemorrhage or non-hemorrhagic neurological deficits, so aggressive treatment is required. ${ }^{1,2)}$ In contrast, DAVFs without CVR are benign, so palliative or conservative treatment is recommended. ${ }^{3)}$ The treatment of DAVFs includes embolization, surgical disconnection, and radiosurgery. Sinus packing through the transvenous approach has a high cure rate for the sinusal type of DAVFs if the affected sinus is not part of the normal venous drainage. Transarterial acrylic glue embolization or surgical disconnection has been performed for the non-sinusal type of DAVFs. Selection of the treatment depends on the location of DAVFs and the

Received June 18, 2014; Accepted November 11, 2014 targeted feeding vessels. For anterior cranial fossa DAVF, glue injection from a branch of the ophthalmic artery carries the risk of visual disturbance caused by glue reflux whereas surgical disconnection of the draining veins is safe. For tentorial DAVF, especially located in the tentorial margin or the vein of Galen, the surgical approach is difficult because of the deep location and complex angioarchitecture, so transarterial glue embolization is performed despite the risk of cranial nerve palsies or glue reflux from the inferolateral trunk (ILT) or meningohypophyseal trunk into the internal carotid artery. ${ }^{4-8)}$ However, large series of cases of transarterial glue embolization are rarely reported despite more than 30-year history of the treatment. ${ }^{9-11)}$ Recently, a new liquid embolic material, ethylene vinyl alcohol copolymer (Onyx, ev3 Neurovascular, Irvine, California, USA) was introduced for the treatment of DAVF and is reported to provide a high cure rate compared to glue embolization. ${ }^{12-19)}$ 
The present study evaluated the efficacy and limitations of transarterial embolization using n-butyl cyanoacrylate (NBCA; Balt Extrusion INC., Montmorency, France) for intracranial DAVFs to investigate the role of NBCA since the introduction of Onyx embolization.

\section{Patients and Methods}

\section{Patients (Table 1)}

In our hospital, sinus packing through the transvenous approach is the standard treatment strategy for sinusal type DAVFs located in the cavernous sinus, transverse-sigmoid sinus (T-SS), and anterior condylar confluence. In contrast, DAVFs located in the anterior cranial fossa are treated by surgical disconnection of the draining veins. Therefore, the indications for transarterial glue embolization are non-sinusal type DAVFs (tentorium, cranial vault), sinusal type DAVFs which require preservation of the affected sinus [Borden type I superior sagittal sinus (SSS) and T-SS DAVFs], access failure by the transvenous approach (Borden type III T-SS DAVFs), and residual shunt after transvenous embolization.

A total of 142 intracranial DAVFs were treated by endovascular therapy between January 2002, when transarterial NBCA embolization was introduced into our hospital, and August 2014. Of these cases, 34 DAVFs in 33 consecutive patients, 22 males and 11 females aged 42 years to 81 years (mean 63.6 years), treated by transarterial NBCA embolization were retrospectively reviewed. The intracranial DAVFs were located in the T-SS in 11 cases, tentorium in 10 , cranial vault in 9 (para SSS in 7, para T-SS in 2 ), and SSS, jugular bulb, foramen magnum, and middle cranial fossa in 1 each. Borden classification was type I in 7 cases, type II in 3, and type III in 24, and CVRs were observed in 27 of the 34 DAVFs $(79 \%)$. The clinical presentations were intracranial hemorrhage in 9 patients, venous infarction in 7 , papilledema due to increased intracranial pressure in 3 , trigeminal neuralgia in 1 , tinnitus in 3 , headache in 2, and incidental discovery in 9. Aggressive lesions containing hemorrhage and non-hemorrhagic neurological deficits were observed in 3 patients with type I (43\%), 1 patient with type II (33\%), and 15 patients with type III DAVFs.

\section{Endovascular treatment}

Transarterial glue embolization was performed under general anesthesia. A 5 or 6 Fr guiding catheter was introduced through the femoral artery. A flow-guided microcatheter $(1.8,1.5$, or 1.2 Fr Magic catheter, Balt, or Marathon catheter, Covidien, Mansfield, Massachusetts, USA) was navigated using a micro- guidewire (SilverSpeed 10 or Mirage 8, Covidien, or GT 12, Terumo, Tokyo) into the feeding arteries as close as possible to the fistula. A $17-67 \%$ mixture of NBCA and Lipiodol (Guerbet LLC., Bloomington, Indiana, USA) was injected under digital subtraction angiography control. After correct penetration of the glue, the microcatheter was rapidly withdrawn. Temporary occlusion balloons (Hyperglide and Hyperform, Covidien, Mansfield, Massachusetts, USA) were used in some cases to prevent reflux into the internal carotid artery. Follow-up angiography was performed within 3 months to 6 months after the treatment. Clinical results were evaluated by the Glasgow outcome scale at discharge or at follow-up outpatient examination. The off-label use of NBCA for DAVF was explained and informed consent was obtained from all patients.

\section{Results}

The treatment and outcomes are summarized in Table 2. Transvenous coil embolization had been previously performed in 8 cases $(7$ T-SS and 1 cranial vault). Residual shunt at the confluence was observed in 1 (T-SS) of the 2 cases of Borden type I, and fistula developed in the adjacent confluence after complete obliteration in the other (T-SS). Residual shunts were observed in all 3 cases (2 T-SS and 1 cranial vault) of Borden type II. The isolated sinuses were inaccessible in the 3 cases (3 T-SS) of Borden type III.

Transarterial glue embolization was performed in 8 cases and 26 cases undergoing initial treatment, using 1 session in 29 cases, 2 sessions in 4 ( 3 tentorium and 1 T-SS), and 4 sessions in 1 (SSS). The target vessels were the middle meningeal artery (MMA) in 26 cases, occipital artery (OA) in 20, ascending pharyngeal artery (APhA) in 7 (coils were used in two cases instead of glue), posterior meningeal artery (PMA) in 5, posterior cerebral artery in 3, superior cerebellar artery in 2, ILT in 2, posterior inferior cerebellar artery in 1, and anterior temporal artery of the middle cerebral artery in 1.

Immediate complete obliteration was achieved in 5 of the 7 cases of Borden type I, but 1 case with recurrence required additional transvenous embolization. Immediate complete obliteration was achieved in 2 ( 2 T-SS) of the 3 cases of Borden type II, and residual shunt in the other (cranial vault) resulted in complete obliteration due to spontaneous thrombosis. Immediate complete obliteration was achieved in 12 of the 24 cases of Borden type III. Six of the 12 cases with residual shunt developed spontaneous thrombosis resulting in complete obliteration, but 1 case with complete obliteration suffered recurrence 


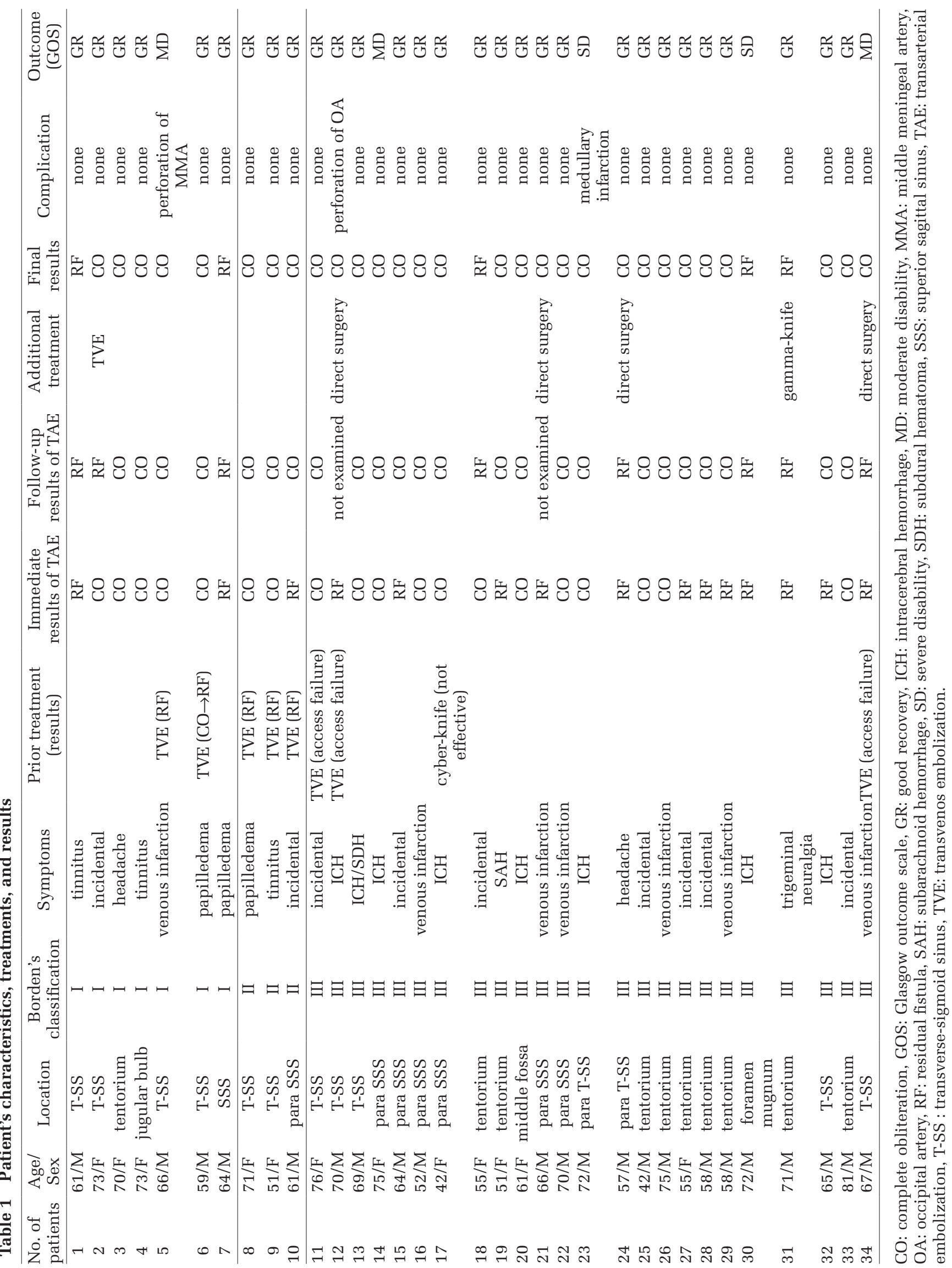

Neurol Med Chir (Tokyo) 55, February, 2015 
Table 2 Results of the treatment

\begin{tabular}{|c|c|c|c|c|}
\hline & \multicolumn{3}{|c|}{ Borden's classification } & \multirow{2}{*}{ Total } \\
\hline & Type I & Type II & Type III & \\
\hline No. of patients & 7 & 3 & 24 & 34 \\
\hline No. of patients undergoing prior TVE & 2 & 3 & 3 & 8 \\
\hline \multicolumn{5}{|l|}{ Results of prior TVE } \\
\hline complete obliteration & 1 & 0 & 0 & 1 \\
\hline residual fistula & 1 & 3 & 0 & 4 \\
\hline access failure & 0 & 0 & 3 & 3 \\
\hline \multicolumn{5}{|l|}{ Complete obliteration after TAE } \\
\hline immediate & $5(71 \%)$ & $2(67 \%)$ & $12(50 \%)$ & $19(56 \%)$ \\
\hline follow-up & $4(57 \%) * 1$ & $3(100 \%)^{* 2}$ & $17(71 \%)^{* 3}$ & $24(71 \%)$ \\
\hline Final complete obliteration & $5(71 \%)^{* 4}$ & $3(100 \%)$ & $21(88 \%)^{* 5}$ & $29(85 \%)$ \\
\hline Complication & 1 & 0 & 2 & $3 * 6(9 \%)$ \\
\hline \multicolumn{5}{|l|}{ Clinical results (GOS) } \\
\hline good recovery & 6 & 3 & 20 & 29 \\
\hline moderate disability & 1 & 0 & 2 & 3 \\
\hline severe disability & 0 & 0 & 2 & 2 \\
\hline
\end{tabular}

${ }^{* 1}$ : 1 recurrence, ${ }^{* 2}: 1$ spontaneous thrombosis, ${ }^{* 3}: 6$ spontaneous thrombosis, 1 recurrence, ${ }^{* 4}: 1$ additional TVE (complete obliteration), ${ }^{* 5}: 4$ additional direct surgery (complete obliteration), 1 additional radiosurgery (residual fistula), ${ }^{* 6}: 2$ perforation (asymptomatic), 1 medullary infarction, GOS: Glasgow outcome scale, TAE: transarterial embolization, TVE: transvenous embolization.

(tentorium). Four cases (2 T-SS and 2 cranial vaults) underwent surgical interruption of the draining veins and complete obliteration was obtained in all cases. One case with tentorial DAVF underwent stereotactic radiosurgery, but the shunt remained patent at 3-year follow-up examination. Overall, complete obliteration rate was $56 \%$ immediately after embolization, $71 \%$ on follow-up angiography, and $85 \%$ after additional treatments. The complete obliteration rates at various concentrations of NBCA were $67 \%$ (6 of 9 cases) using low concentration (17-20\% NBCA), $73 \%$ (16 of 22 cases) using moderate concentration (25-40\% NBCA), and $67 \%$ ( 2 of 3 cases) using high concentration (50-67\% NBCA).

Complications occurred in three patients. Two patients suffered asymptomatic vessel perforations during cannulation and the other patient had leakage of contrast medium resulting in medullary infarction. Cranial nerve palsy or venous infarction and/ or hemorrhage resulting from excessive migration of the glue into the draining veins did not occur. Glasgow outcome scale was good recovery in 29, moderate disability in 3 , and severe disability in 2 .

\section{Representative Cases}

Case 10: A 61-year-old man was incidentally discovered para SSS DAVF by digital subtraction angiography performed for right occipital lobe arteriovenous malfor- mation. Neurological examination demonstrated left homogeneous hemianopia due to right occipital lobe arteriovenous malformation. Right external carotid angiography showed the DAVF fed by the superficial temporal artery and MMA, and draining into the cortical veins and SSS (Fig. 1A). Right occipital artery angiography (Fig. 1B) and the multi-planar reconstruction image of computed tomography (CT) angiography (Fig. 1C) showed the fistulous pouch in an intraosseous location. Embolization was performed under general anesthesia. The antero-posterior view (caudal projection; Fig. 1D) of right external carotid angiography showed a connection (arrow) between the draining vein and the SSS. A microcatheter was introduced into the draining vein via the SSS through a transvenous route, but could not be passed into the venous pouch (Fig. 1E). The draining veins were embolized using coils, but the shunt remained patent (Fig. 1F). Then, 33\% NBCA was injected from the posterior branch of the MMA, but did not reach the fistulous pouch (Fig. 1G). Right external carotid angiography showed a residual shunt (Fig. 1H). Four months after the embolization, the residual shunt had completely disappeared (Fig. 1I).

Case 11: A 76-year-old woman was incidentally discovered to have a T-SS DAVF by magnetic resonance (MR) imaging performed after head injury. Left external carotid angiography showed a DAVF with isolated left T-SS fed by the MMA, OA, and APhA, 

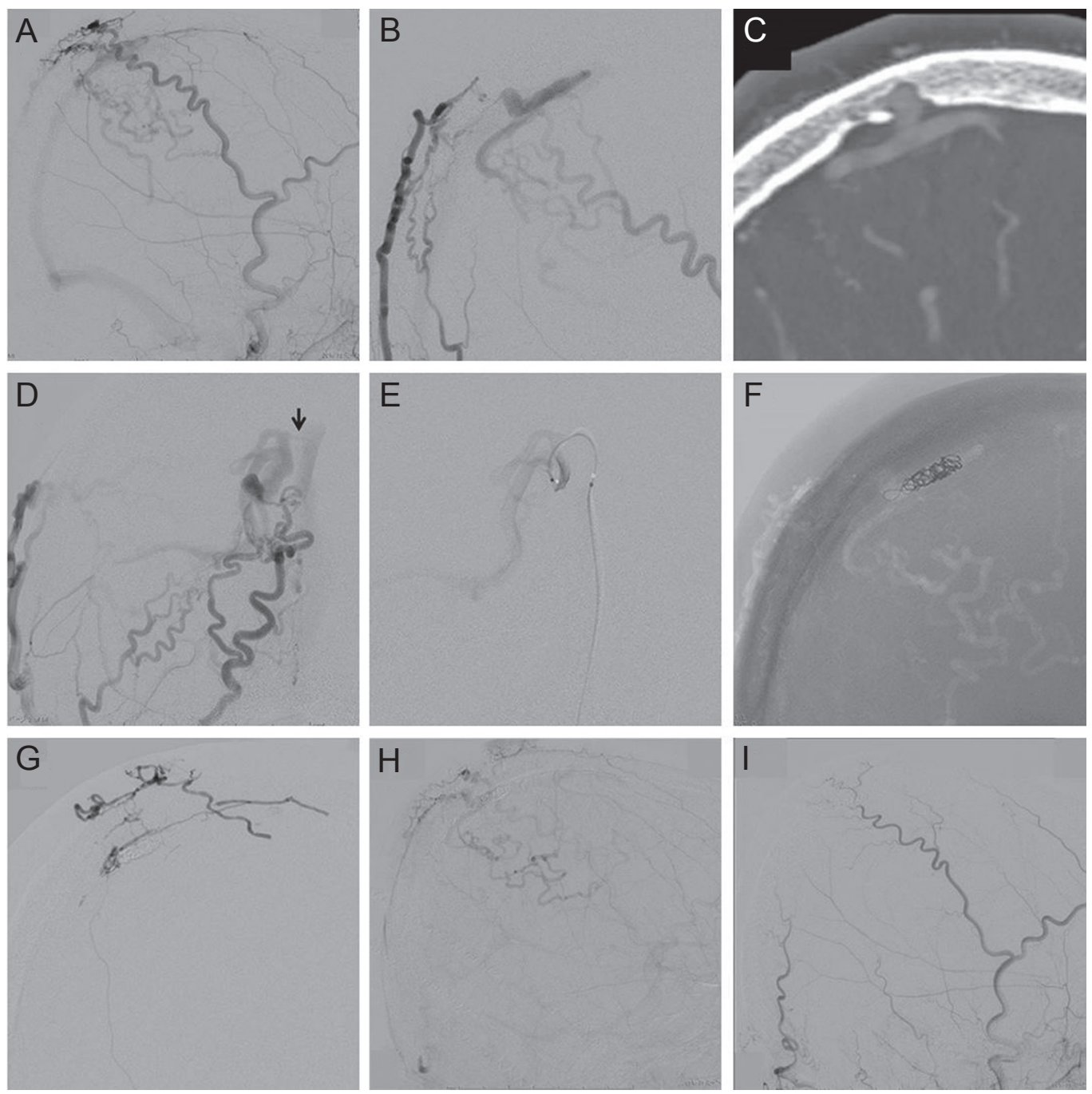

Fig. 1 (Case 10) A 61-year-old man with incidentally discovered cranial vault dural arteriovenous fistula (DAVF). A: Right external carotid angiogram showing a DAVF fed by the superficial temporal artery and middle meningeal artery, and draining into the cortical veins and superior sagittal sinus (SSS). B, C: Right occipital artery angiogram (B) and multi-planar reconstruction image of computed tomography angiography (C) showing an intraosseous location of the fistulous pouch. D: Antero-posterior view (caudal projection) of right external carotid angiogram showing a connection (arrow) between the draining vein and the SSS. E: A microcatheter was introduced into the draining vein via the SSS through a transvenous route, but could not be passed into the venous pouch. F: The draining veins were embolized using coils. G: $33 \%$ n-Butyl cyanoacrylate was injected from the posterior branch of the middle meningeal artery, but did not reach the fistulous pouch. H: Right external carotid angiogram showing a residual shunt. I: Four months after the embolization, the residual shunt had completely disappeared.

and draining into the parietal cortical veins (Fig. 2A). Transvenous embolization was attempted, but the microcatheter could not be advanced into the isolated sinus. After glue embolization of the left OA, a microcatheter was introduced into the MMA. Warm 20\% NBCA was injected and penetrated into the isolated sinus (Fig. 2B), but was rapidly washed away into the draining veins (Fig. 2C). She had no symptoms. Left external carotid angiography after embolization showed complete obliteration (Fig. 2D). Case 23: A-72-year-old man, who underwent surgery for left cerebellar hemangioblastoma 21 years before, and suffered left hemi-ataxia, was discovered to have right temporo-occipital lobe hematoma by CT 13 days after irrigation surgery for right chronic subdural hematoma. Cerebral angiography showed a DAVF adjacent to the right T-SS, which was fed by the right MMA and PMA and drained into the occipital cortical vein (Fig. 3A), and transarterial embolization was performed. During cannulation to the right PMA, leakage of the contrast medium occurred (Fig. 3B) and 50\% NBCA was immediately 

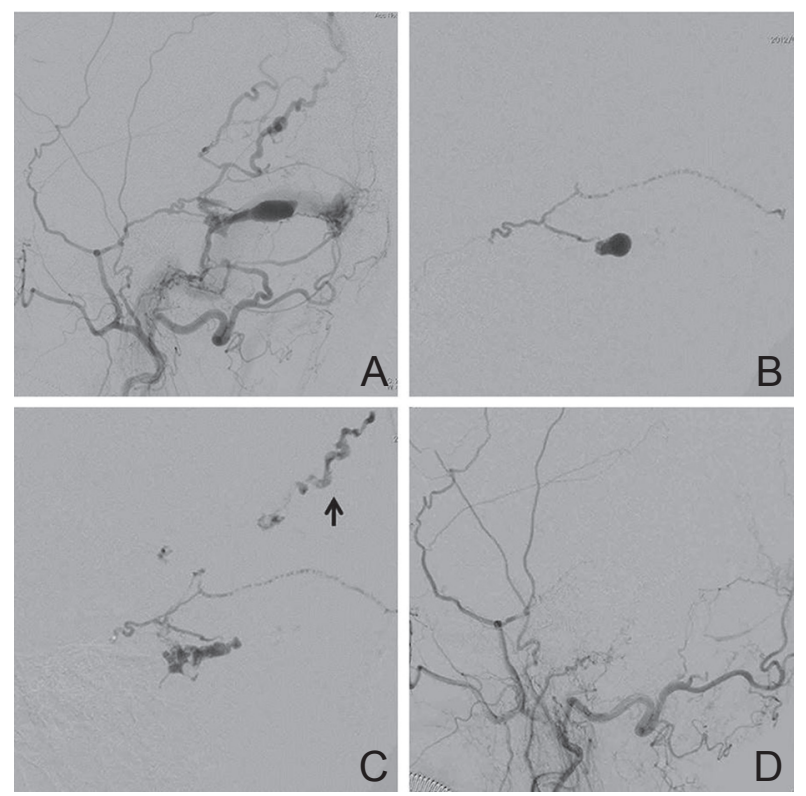

Fig. 2 (Case 11) A 76-year-old woman with transversesigmoid sinus (T-SS) dural arteriovenous fistula (DAVF) identified by magnetic resonance imaging performed after head injury. A: Left external carotid angiogram showing a DAVF with isolated left T-SS fed by the middle meningeal artery, occipital artery, and ascending pharyngeal artery, draining into the parietal cortical veins. B, C: A microcatheter was introduced into the middle meningeal artery. Warm $20 \%$ n-butyl cyanoacrylate penetrated into the isolated sinus (B), but was rapidly washed away into the draining veins (arrow) (C). D: Left external carotid angiogram after embolization showing complete obliteration.
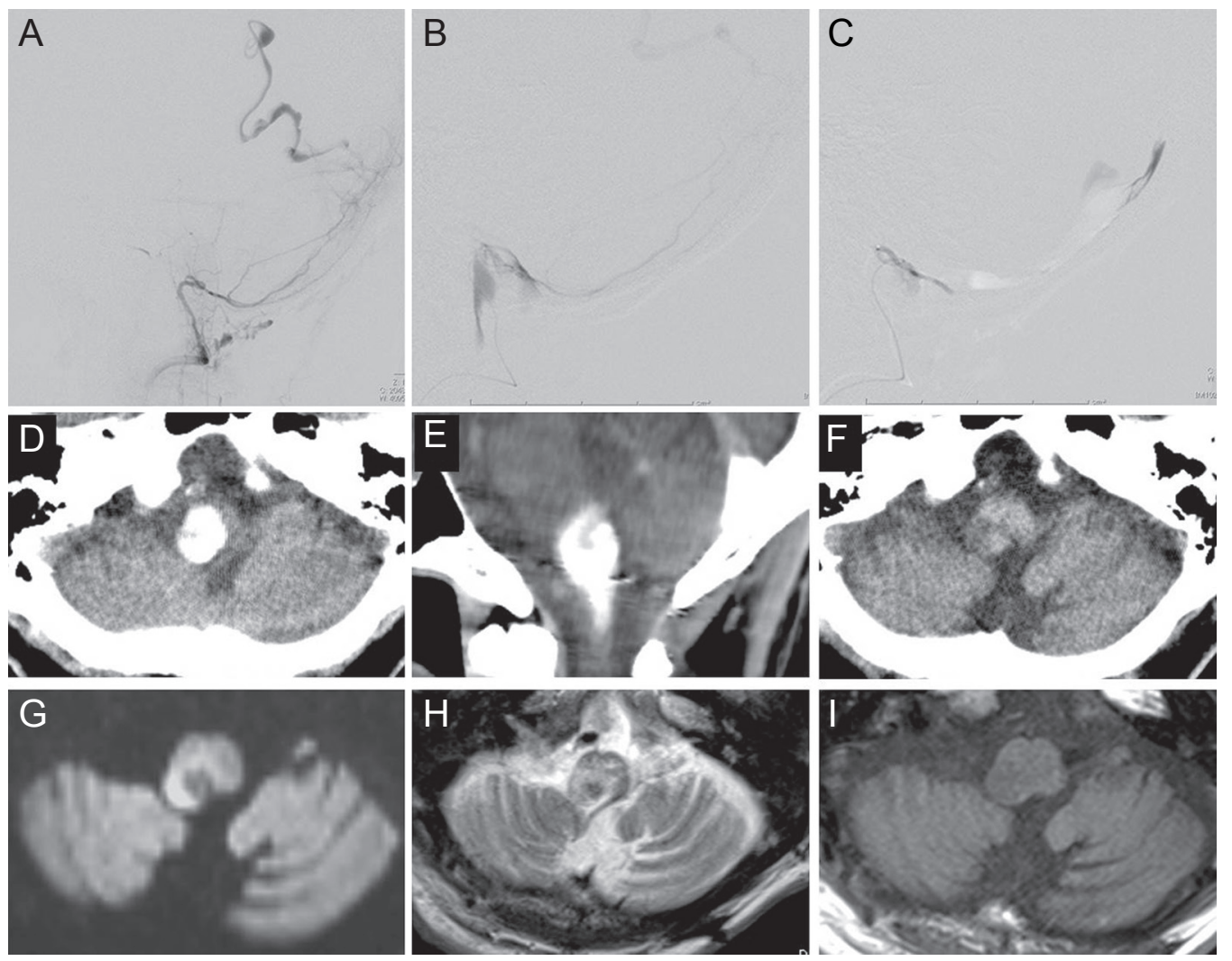

Fig. 3 (Case 23) A 72-year-old man with right para transverse-sigmoid sinus (T-SS) dural arteriovenous fistula (DAVF) presenting with temporo-occipital lobe hematoma. A: Right vertebral angiogram showing a right para T-SS DAVF, fed by posterior meningeal artery and drained into the occipital cortical vein. B: Leakage of the contrast medium occurred during a cannulation to the right posterior meningeal artery. C: 50\% n-Butyl cyanoacrylate was immediately injected. D, E: Computed tomography (CT) scans performed immediately after embolization demonstrating a high density area at the left side of the medulla. F: CT scan taken on the day after embolization showing disappearance of the high density area. G: Diffusion-weighted magnetic resonance (MR) image on the day after embolization demonstrating a high intensity area at the left postero-lateral medulla $\mathrm{H}$, I: The lesion identified on diffusion-weighted MR image was delineated as a high intensity area with low intensity area in the central portion on $T_{2}$-weighted $M R$ image $(H)$ and a low intensity area on $T_{1}$-weighted MR image (I). 
injected (Fig. 3C). Then, NBCA embolization from the left MMA was performed and complete obliteration was obtained. CT performed immediately after embolization demonstrated a high density area at the left side of the medulla (Fig. 3D, E), but this had disappeared by the next day (Fig. 3F). Diffusionweighted MR imaging demonstrated a high intensity area suggesting infarction at the left postero-lateral medulla (Fig. 3G). This lesion was delineated as a high intensity area with low intensity area in the central portion on $\mathrm{T}_{2}$-weighted imaging (Fig. $3 \mathrm{H}$ ) and low intensity area on $\mathrm{T}_{1}$-weighted imaging (Fig. 3I). He had persistent bilateral hemiataxia, dysphagia, and dysarthria.

Case 31: A 71-year-old man was referred to our hospital for a 3-year history of left trigeminal neuralgia. Cerebral angiography showed a DAVF at the left tentorial margin, fed by the left ILT and drained into the petrosal veins with a varix that was responsible for the trigeminal neuralgia (Fig. 4A).
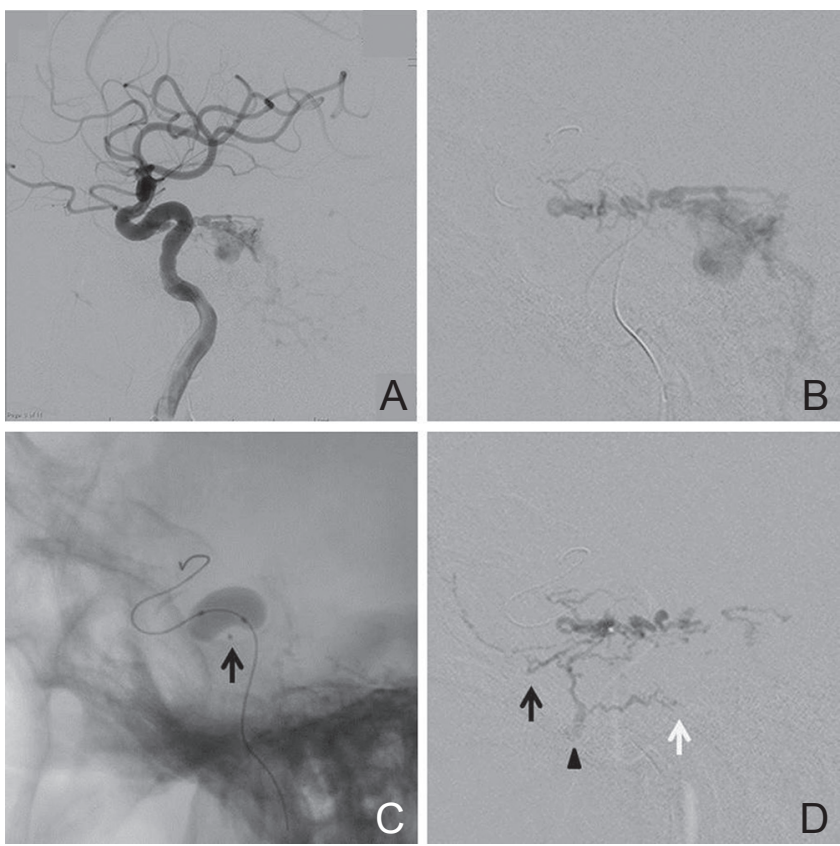

Fig. 4 (Case 31) A 71-year-old man with a tentorial margin dural arteriovenous fistula (DAVF) presenting with trigeminal neuralgia. A: Left internal carotid angiogram showing a DAVF fed by the inferolateral trunk (ILT) and draining into the dilated petrosal vein. B: A microcatheter was introduced into the ILT. C: The balloon was inflated at the origin of the ILT to prevent glue reflux into the internal carotid artery. Arrow indicates the tip of the microcatheter. D: $33 \%$ n-Butyl cyanoacrylate was injected during balloon inflation. The glue penetrated into the artery of foramen rotundum (arrow), accessory meningeal artery (arrowhead), and vidian artery (white arrow), but did not penetrate into the dilated petrosal vein.
The DAVF was also fed by the left APhA, MMA, accessory meningeal artery, artery of the foramen rotundum, OA, and the right meningohypophyseal trunk. Transarterial embolization was performed under general anesthesia. After glue embolization of the left MMA, a microcatheter was introduced into the left ILT (Fig. 4B). A Hyperform occlusion balloon was inflated at the origin of the ILT to prevent glue reflux into the internal carotid artery (Fig. 4C), and $33 \%$ NBCA was injected during balloon inflation. The glue penetrated into the artery of foramen rotundum, accessory meningeal artery, and vidian artery, but did not penetrate into the dilated petrosal vein (Fig. 4D). The shunts from the left internal carotid artery disappeared, but remained patent from the left OA and right meningohypophyseal trunk. After embolization, his facial pain disappeared according to the reduction of the dilated petrosal vein. $\mathrm{He}$ underwent second embolization for left $\mathrm{OA}$ and radiosurgery for the residual shunt, but complete obliteration could not be obtained.

\section{Discussion}

The angiographic cure rate of glue embolization ranges widely from $29.8 \%$ to $100 \% .^{9-11)}$ These differences are considered to derive from variations in the technique and the patient selection, suggesting that glue can achieve high cure rates if used under the correct technical and anatomical conditions. ${ }^{9)}$ Onyx is a non-adhesive liquid embolic material recently introduced for the treatment of DAVFs, which allows prolonged injection and good penetration into the draining veins under well-controlled conditions, with consequent high cure rates $(82-100 \%) .{ }^{12-19)}$ The initial angiographic occlusion rate of Onyx embolization was significantly higher (29/35) than that of NBCA embolization (7/21), and fewer patients have required subsequent surgery (5/35) compared to NBCA $(11 / 21){ }^{20)}$

In our series, the immediate and follow-up complete obliteration rates in all cases were $56 \%$ and $71 \%$ (52\% and $66 \%$ in 29 cases treated with only transarterial glue embolization). Seven of the 13 patients with residual shunts showed complete obliteration due to spontaneous thrombosis, whereas 2 patients suffered recurrence after complete occlusion. The high thrombogenicity of glue can lead to complete obliteration even if residual shunts are present.

Various techniques to increase glue penetration into the draining veins have been reported: the catheter weadged technique, ${ }^{11)}$ low concentrations of glue, ${ }^{21)}$ the use of balloons, ${ }^{8}$ and the simultaneous injection of glue and glucose. ${ }^{22)}$ Low concentration glue injected from the wedged microcatheter can 
penetrate other feeders and draining veins similar to Onyx (Case 10, Fig. 1G).

Transarterial glue embolization is effective for Borden type III DAVFs with direct CVR. However, Borden type III DAVFs that drain into the dural sinus with secondary CVR as seen in T-SS DAVFs are sometimes difficult to close because of the low penetration of the glue. For such cases, we prefer transvenous embolization using coils. In our hospital, 13 of 15 patients with isolated T-SS DAVFs were initially treated with the transvenous approach and the isolated sinus was accessible in 10 patients, resulting in complete cure in 8 patients. Transarterial glue embolization is effective for small residual shunts persisting after transvenous embolization.

Transarterial glue embolization has limitations to treat Borden type I and II DAVFs. ${ }^{10)}$ Onyx embolization has advantages for such cases despite other limitations. ${ }^{12,23,24)}$ Complete cure was achieved with preserved patency of the sinus in 7 of 20 patients with Borden type I and type II DAVFs in the T-SS and SSS. ${ }^{12)}$ Two patients treated with transarterial Onyx embolization had preserved patency of the sinus by inflating a balloon in the patent sinus via the venous approach. ${ }^{25)}$ Transarterial Onyx embolization is now becoming the first-line treatment even for Borden type I and II sinusal type DAVFs.

Complications of glue embolization include vessel perforation, glue migration into the cerebral arteries, cranial nerve palsies, and excessive penetration into the draining veins resulting in venous infarction. In our series, asymptomatic vessel perforations occurred in two patients and leakage of the contrast medium in one patient (Case 23, Fig. 3G) during cannulation. We assume that the injection of contrast medium from the microcatheter wedged into the perforating artery to the medulla resulted in leakage of the contrast medium, which was misinterpreted as vessel perforation, and NBCA injection to stop bleeding resulted in medullary infarction. Therefore, gentle maneuvering is essential during introduction of the microcatheter into tortuous and narrow feeding arteries.

Glue migration into the cerebral arteries may occur as a result of reflux into the targeted vessels or penetration into other feeding vessels through the arterial network between the feeders. The latter can be prevented by precise understanding of the angioarchitecture and prediction of the behavior of the injected glue. The former can be avoided by distal navigation of the microcatheter or use of a balloon $^{19)}$ if distal navigation of the microcatheter is not possible (Case 31, Fig. 4C). Care must be taken to avoid rupture of a latex balloon caused by contact with Lipiodol or by friction between the balloon and the microcatheter during pullout of the microcatheter.

In the present series, no cranial nerve palsies occurred despite glue injection from the APhA, the ILT, and the petrosal branch of the MMA supplying the cranial nerves. We believe that NBCA hardly permeates into the vasa nervosa because of its adherence and the viscosity of the mixture with Lipiodol unless the injection is forced from the wedged microcatheter. Cranial nerve palsies are apparently more common after Onyx embolization than glue embolization because of the high penetration of Onyx and the angiotoxic effect of dimethyl sulfoxide used as a solvent. ${ }^{26)}$ Distal navigation of the microcatheter beyond the foramina where the cranial nerves pass is necessary to avoid cranial nerve palsies. NBCA was injected with careful attention to the reflux in a T-SS DAVF fed by the hypoglossal branch of the APhA, because the microcatheter could be advanced beyond the hypoglossal canal (Case 32, Fig. 5A-C). In contrast, proximal occlusion using coils was performed in a T-SS DAVF fed by jugular branches of the APhA, because distal navigation of the microcatheter beyond the jugular foramen was impossible (Case 34, Fig. 5D-F).

Extensive glue penetration into the draining veins may cause venous infarction or hemorrhage, although effective embolization requires glue penetration into the draining veins. ${ }^{10,27)}$ Special care is required if deep venous drainage is involved in the DAVFs, as in tentorial DAVFs. ${ }^{5,13)}$ Glue passing through the fistula is fragmented and rapidly washed away into the draining veins by the stream of other feeders in high flow DAVFs (Case 11, Fig. 2C). In such cases, devascularization of the high-flow shunts is required and high concentration glue can be used for flow control before definitive glue injection. If coils are placed in the draining veins or the sinus through the transvenous or transarterial approach, in rare cases the microcatheter can be advanced transarterially into the venous side beyond the fistula, ${ }^{6)}$ and the adherent property of the glue can prevent excessive glue migration into the draining veins. Unexpected migration of Onyx into the draining veins, lungs, and heart has been reported. ${ }^{24,27)}$ Although the plug and push technique can minimize this risk, Onyx seems to be unsuitable for occlusion of high flow shunt because precipitation takes time.

The benefits of transarterial glue embolization compared to Onyx are high thrombogenicity leading to complete obliteration if residual shunts are present, relatively low risks of cranial nerve palsies if injected from the feeding arteries supplying the cranial nerves and of excessive migration into the draining veins in high flow shunts, and high 

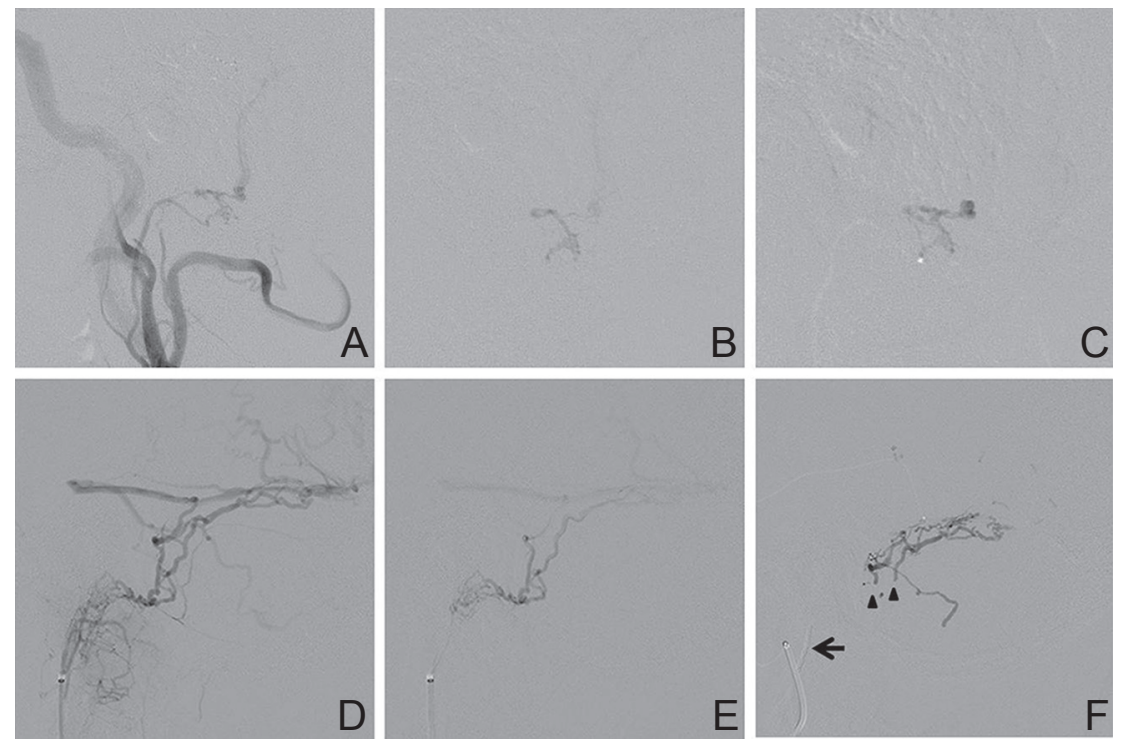

Fig. 5 Embolization from the ascending pharyngeal artery (A-C, Case 32; D-F, Case 34) A: Left external carotid angiogram showing a left transverse-sigmoid sinus dural arteriovenous fistula (DAVF) fed by the hypoglossal branch of ascending pharyngeal artery (APhA). B: A microcatheter was advanced beyond the hypoglossal canal. C: $33 \%$ n-Butyl cyanoacrylate (NBCA) was injected without reflux. D: Left external carotid angiogram showing a left transverse-sigmoid sinus DAVF fed by the jugular branch of APhA. E: Distal navigation of the microcatheter beyond the jugular foramen was impossible because multiple small branches fed the DAVF, then the APhA was embolized using coils. F: 17\% NBCA injected from the left middle meningeal artery penetrated into the feeders from the APhA (arrowheads). Arrow indicates the coils deployed in the APhA.

compatibility with CT, MR imaging, and digital subtraction angiography for follow-up examinations. ${ }^{28)}$ However, the behavior of injected glue is sometimes unpredictable and glue embolization, especially from the ILT or APhA, carries a high risk of cerebral infarction or cranial nerve palsies, so precise understanding of the angioarchitecture and proficiency in the handling of NBCA are essential.

Transarterial glue embolization continues to be useful for selected patients, and complete cure can be expected in most patients with fewer complications if combined with transvenous embolization or direct surgery. ${ }^{29)}$

\section{Conclusion}

Transarterial glue embolization is highly effective for Borden type III DAVFs with direct CVR, but has limitations for Borden type I and II DAVFs with diffuse shunts in which the affected sinus cannot be sacrificed. The understanding of the differences between Onyx and NBCA is important in the selection of embolic materials.

\section{Conflicts of Interest Disclosure}

The authors have no personal, financial, or insti- tutional interest in any of the drugs, materials, or devices in the article. All authors who are members of The Japan Neurosurgical Society (JNS) have registered online Self-reported COI Disclosure Statement Forms through the website for JNS members.

\section{References}

1) Borden JA, Wu JK, Shucart WA: A proposed classification for spinal and cranial dural arteriovenous fistulous malformations and implications for treatment. J Neurosurg 82: 166-179, 1995

2) Cognard C, Gobin YP, Pierot L, Bailly AL, Houdart E, Casasco A, Chiras J, Merland JJ: Cerebral dural arteriovenous fistulas: clinical and angiographic correlation with a revised classification of venous drainage. Radiology 194: 671-680, 1995

3) Satomi J, van Dijk JM, Terbrugge KG, Willinsky RA, Wallace MC: Benign cranial dural arteriovenous fistulas: outcome of conservative management based on the natural history of the lesion. $J$ Neurosurg 97: 767-770, 2002

4) Huang Q, Xu Y, Hong B, Li Q, Zhao W, Liu J: Use of onyx in the management of tentorial dural arteriovenous fistulae. Neurosurgery 65: 287-292; discussion 292-293, 2009

5) Jiang C, Lv X, Li Y, Zhang J, Wu Z: Endovascular treatment of high-risk tentorial dural arteriovenous 
fistulas: clinical outcomes. Neuroradiology 51: 103-111, 2009

6) Miyamoto N, Naito I, Takatama S, Iwai T, Matsumoto M, Kawauchi H, Nishigaya K, Shimaguchi H, Shimizu T, Yoshimoto Y: [Endovascular treatment for tentorial dural arteriovenous fistulas]. Surgery for Cerebral Stroke 38: 235-242, 2010 (Japanese)

7) Puffer RC, Daniels DJ, Kallmes DF, Cloft HJ, Lanzino G: Curative Onyx embolization of tentorial dural arteriovenous fistulas. Neurosurg Focus 32: E4, 2012

8) van Rooij WJ, Sluzewski M, Beute GN: Tentorial artery embolization in tentorial dural arteriovenous fistulas. Neuroradiology 48: 737-743, 2006

9) Guedin P, Gaillard S, Boulin A, Condette-Auliac S, Bourdain F, Guieu S, Dupuy M, Rodesch G: Therapeutic management of intracranial dural arteriovenous shunts with leptomeningeal venous drainage: report of 53 consecutive patients with emphasis on transarterial embolization with acrylic glue. J Neurosurg 112: 603-610, 2010

10) Kim DJ, Willinsky RA, Krings T, Agid R, Terbrugge K: Intracranial dural arteriovenous shunts: transarterial glue embolization-experience in 115 consecutive patients. Radiology 258: 554-561, 2011

11) Nelson PK, Russell SM, Woo HH, Alastra AJ, Vidovich DV: Use of a wedged microcatheter for curative transarterial embolization of complex intracranial dural arteriovenous fistulas: indications, endovascular technique, and outcome in 21 patients. J Neurosurg 98: 498-506, 2003

12) Abud TG, Nguyen A, Saint-Maurice JP, Abud DG, Bresson D, Chiumarulo L, Enesi E, Houdart E: The use of Onyx in different types of intracranial dural arteriovenous fistula. AJNR Am J Neuroradiol 32: 2185-2191, 2011

13) Cognard C, Januel AC, Silva NA, Tall P: Endovascular treatment of intracranial dural arteriovenous fistulas with cortical venous drainage: new management using Onyx. AJNR Am J Neuroradiol 29: 235-241, 2008

14) $\mathrm{Hu} Y C$, Newman CB, Dashti SR, Albuquerque FC, McDougall CG: Cranial dural arteriovenous fistula: transarterial Onyx embolization experience and technical nuances. J Neurointerv Surg 3: 5-13, 2011

15) De Keukeleire K, Vanlangenhove P, Kalala Okito JP, Hallaert G, Van Roost D, Defreyne L: Transarterial embolization with ONYX for treatment of intracranial non-cavernous dural arteriovenous fistula with or without cortical venous reflux. J Neurointerv Surg 3: 224-228, 2011

16) Maimon S, Nossek E, Strauss I, Blumenthal D, Frolov V, Ram Z: Transarterial treatment with Onyx of intracranial dural arteriovenous fistula with cortical drainage in 17 patients. AJNR Am J Neuroradiol 32: 2180-2184, 2011

17) Nogueira RG, Dabus G, Rabinov JD, Eskey CJ, Ogilvy CS, Hirsch JA, Pryor JC: Preliminary experience with onyx embolization for the treatment of intracranial dural arteriovenous fistulas. AJNR Am J Neuroradiol 29: 91-97, 2008

18) Rangel-Castilla L, Barber SM, Klucznik R, Diaz O: Mid and long term outcomes of dural arteriovenous fistula endovascular management with Onyx. Experience of a single tertiary center. J Neurointerv Surg 6: 607-613, 2014

19) van Rooij WJ, Sluzewski M: Curative embolization with Onyx of dural arteriovenous fistulas with cortical venous drainage. AJNR Am J Neuroradiol 31: 1516-1520, 2010

20) Rabinov JD, Yoo AJ, Ogilvy CS, Carter BS, Hirsch JA: ONYX versus n-BCA for embolization of cranial dural arteriovenous fistulas. J Neurointerv Surg 5: 306-310, 2013

21) Li MH, Tan HQ, Fang C, Zhu YQ, Wang W, Wang J, Cheng YS: Trans-arterial embolisation therapy of dural carotid-cavernous fistulae using low concentration n-butyl-cyanoacrylate. Acta Neurochir (Wien) 150: 1149-1156; discussion 1156, 2008

22) Moore C, Murphy K, Gailloud P: Improved distal distribution of $n$-butyl cyanoacrylate glue by simultaneous injection of dextrose $5 \%$ through the guiding catheter: technical note. Neuroradiology 48: 327-332, 2006

23) Jiang C, Lv X, Li Y, Wu Z: Transarterial Onyx packing of the transverse-sigmoid sinus for dural arteriovenous fistulas. Eur J Radiol 80: 767-770, 2011

24) Lv X, Jiang C, Li Y, Liu L, Liu J, Wu Z: The limitations and risks of transarterial Onyx injections in the treatment of grade I and II DAVFs. Eur J Radiol 80: e385-e388, 2011

25) Jittapiromsak P, Ikka L, Benachour N, Spelle L, Moret J: Transvenous balloon-assisted transarterial Onyx embolization of transverse-sigmoid dural arteriovenous malformation. Neuroradiology 55: 345-350, 2013

26) Elhammady MS, Wolfe SQ, Farhat H, Moftakhar R, Aziz-Sultan MA: Onyx embolization of carotidcavernous fistulas. J Neurosurg 112: 589-594, 2010

27) Wang H, Lv X, Jiang C, Li Y, Wu Z, Xu K: Onyx migration in the endovascular management of intracranial dural arteriovenous fistulas. Interv Neuroradiol 15: 301-308, 2009

28) Adamczyk P, Amar AP, Mack WJ, Larsen DW: Recurrence of "cured" dural arteriovenous fistulas after Onyx embolization. Neurosurg Focus 32(5): E12, 2012

29) Natarajan SK, Ghodke B, Kim LJ, Hallam DK, Britz GW, Sekhar LN: Multimodality treatment of intracranial dural arteriovenous fistulas in the Onyx era: a single center experience. World Neurosurg 73: 365-379, 2010

Address reprint requests to: Naoko Miyamoto, MD, Department of Neurosurgery, Geriatrics Research Institute and Hospital, 3-26-8 Ootomo-cho, Maebashi, Gunma 371-0847, Japan. e-mail: naochop96201088@yahoo.co.jp 